\title{
Low-Dose CT for Craniosynostosis: Preserving Diagnostic Benefit with Substantial Radiation Dose Reduction
}

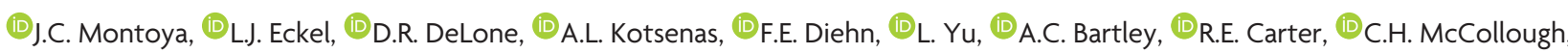 \\ and $\oplus_{\text {J.G. Fletcher }}$
}

\begin{abstract}
BACKGROUND AND PURPOSE: Given the positive impact of early intervention for craniosynostosis, CT is often performed for evaluation but radiation dosage remains a concern. We evaluated the potential for substantial radiation dose reduction in pediatric patients with suspected craniosynostosis.
\end{abstract}

MATERIALS AND METHODS: CT projection data from pediatric patients undergoing head CT for suspected craniosynostosis were archived. Simulated lower-dose CT images corresponding to $25 \%, 10 \%$, and $2 \%$ of the applied dose were created using a validated method. Three neuroradiologists independently interpreted images in a blinded, randomized fashion. All sutures were evaluated by using 3D volume-rendered images alone, and subsequently with 2D and 3D images together. Reference standards were defined by reader agreement by using routine dose and 2D and 3D images. Performance figures of merit were calculated based on reader response and confidence.

RESULTS: Of 33 pediatric patients, 21 had craniosynostosis (39 positive sutures and 225 negative sutures). The mean volume CT dose index was $15.5 \pm 2.3 \mathrm{mGy}$ (range, 9.69-19.38 mGy) for the routine dose examination. Average figures of merit for multireader analysis ranged from $0.92(95 \% \mathrm{Cl}, 0.90-0.95)$ at routine pediatric dose to $0.86(95 \% \mathrm{Cl}, 0.79-0.94)$ at $2 \%$ dose using 3D images alone. Similarly, pooled reader figures of merit ranged from $0.91(95 \% \mathrm{Cl}, 0.89-0.95)$ at routine pediatric dose to $0.85(95 \% \mathrm{Cl}, 0.76-0.95)$ at $2 \%$ dose using $2 \mathrm{D}$ and $3 \mathrm{D}$ images together. At $25 \%$ and $10 \%$ dose, $95 \% \mathrm{Cl}$ of the difference in figures of merit from routine dose included 0 , suggesting similar or noninferior performance.

CONCLUSIONS: For pediatric head CT for evaluation of craniosynostosis, dose reductions of $75 \%-90 \%$ were possible without compromising observer performance.

ABBREVIATIONS: $\mathrm{CTDI}_{\mathrm{vol}}=$ volume $\mathrm{CT}$ dose index; $\mathrm{FOM}=$ figure of merit

D eformities of the skull in infants and young children pose a diagnostic challenge to the examining medical provider. The challenges of differentiating benign deformational plagiocephaly from craniosynostosis often require imaging. ${ }^{1}$ Because untreated craniosynostosis can inhibit brain development and raise intracranial pressure, because the skulls do not expand enough to make room for the growing brain, a thorough evaluation should be performed on all children with suspected craniosynostosis. In

Received July 6, 2016; accepted after revision November 6.

From the Departments of Radiology (J.C.M., L.J.E., D.R.D., A.L.K., F.E.D., L.Y., C.H.M., J.G.F.) and Health Sciences Research (A.C.B., R.E.C.), Mayo Clinic, Rochester, Minnesota.

Support for this study was provided in part by Siemens Healthcare through a research grant awarded to Dr. Cynthia H. McCollough.

Paper previously presented in part at: Annual Meeting of the Radiological Society of North America, November 29 to December 4, 2015; Chicago, Illinois.

Please address correspondence to Joel G. Fletcher, MD, Department of Radiology, Mayo Clinic, 200 First St SW, Rochester, MN 55905; e-mail: fletcher.joel@mayo.edu

http://dx.doi.org/10.3174/ajnr.A5063 mild cases of craniosynostosis, no treatment may be required, or a specially molded helmet may be used to reshape the baby's head and allow room for normal brain growth. ${ }^{2}$

Although a complete evaluation includes a detailed pregnancy, birth, and sleeping position history, as well as a thorough physical examination, imaging is often needed to confirm the diagnosis. CT is the preferred imaging technique for accurate and complete assessment. ${ }^{1}$ However, because CT uses ionizing radiation in the acquisition of images, some have recommended deferral of CT when the diagnosis is less than certain clinically, ${ }^{3}$ even though the risk associated with the radiation doses used in CT is too low to be convincingly demonstrated. ${ }^{4}$ Still, some physicians and parents are concerned about the radiation dose used in CT. Therefore, given the positive impact of early intervention, it is important to minimize radiation dose and confirm the diagnostic performance of low-dose examinations ${ }^{5}$ to minimize the concerns of parents and some medical professionals about radiation dose, which may result in deferral of CT and, thereby, diagnostic 


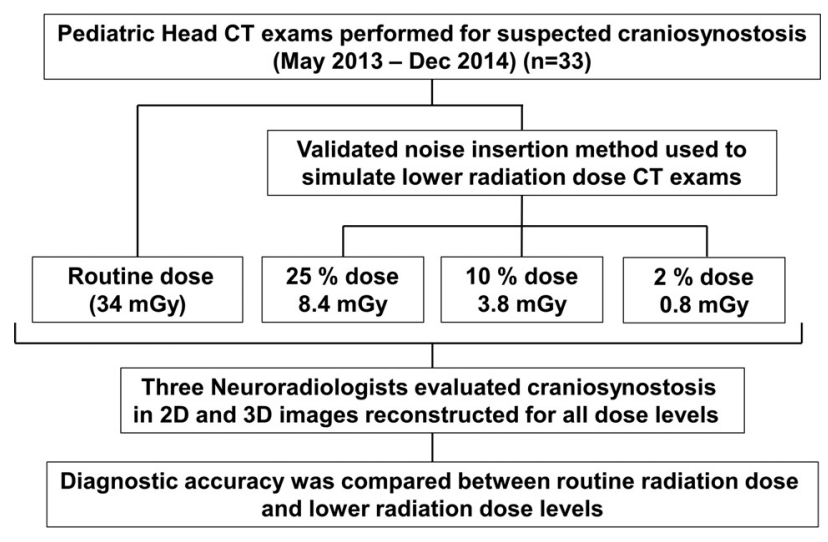

FIG 1. Study schema.

delay. Because osseous tissue is the primary anatomy of interest in imaging for craniosynostosis and evaluation of osseous structures is a high-contrast diagnostic task, assessment of craniosynostosis does not require the same level of image quality as a routine head CT examination, which must also evaluate soft tissue structures. Therefore, radiation dose reduction should be achievable. Iterative reconstruction is a promising approach to reducing CT radiation dose while not hampering diagnostic performance for highcontrast tasks. $^{6}$

The purpose of this study was to determine the minimum radiation dose required to produce clinically acceptable performance for the diagnostic evaluation of craniosynostosis. As part of this evaluation, we used iterative reconstruction to facilitate radiation dose reduction and examined whether the use of $3 \mathrm{D}$ volume-rendered images could improve confidence in interpretation without compromising diagnostic performance.

\section{MATERIALS AND METHODS}

This HIPAA-compliant study was approved by our institutional review board. Selection of cases for inclusion in this study was consistent with Minnesota Research Authorization (Minnesota Statute 144.295). The requirement for written informed consent was waived for this retrospective study. All examinations in this study were performed using our clinical standard of care.

\section{Patient Population}

Thirty-three pediatric patients who underwent unenhanced CT of the head with an indication for craniosynostosis between May 2013 and December 2014 were included in the study. Inclusion criteria included 1) patients $<18$ years of age, 2) who underwent unenhanced CT of the head, 3) by using a 128-section CT system, 4) with an indication for craniosynostosis, and 5) only patients with archived CT projection data were considered. Five cases in our study cohort were scanned after surgery for a question of recurrent or residual craniosynostosis. Figure 1 illustrates our study schema.

\section{Image Acquisition}

Patients were scanned according to our institution's routine pediatric head CT protocol by using a 128-section CT system (Somatom Definition Flash; Siemens, Erlangen, Germany). All

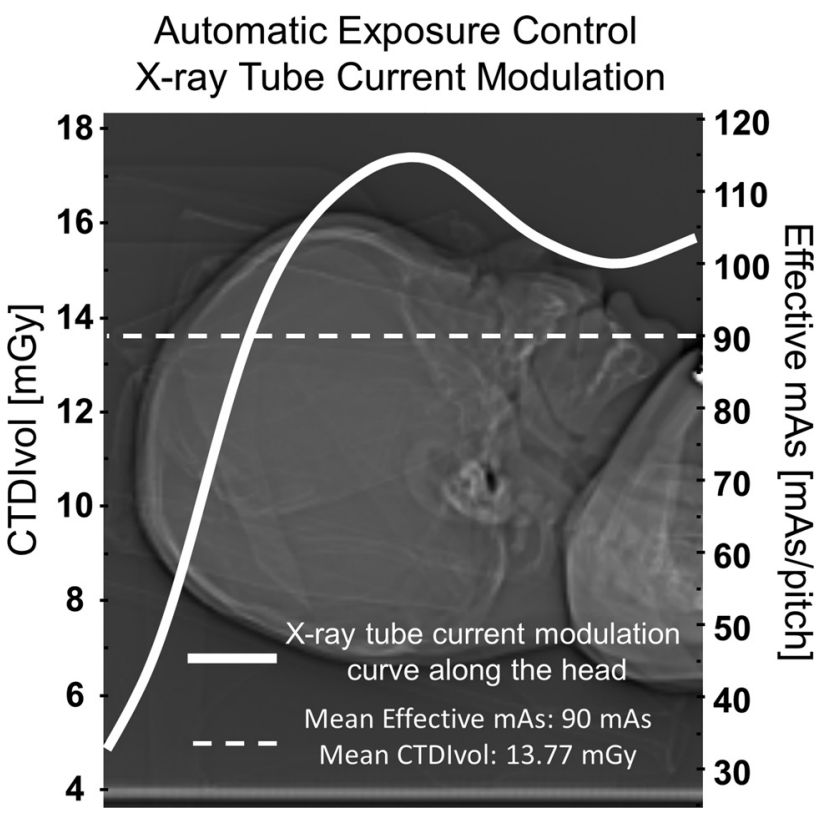

FIG 2. Automatic exposure control in the head of a pediatric patient. The quality reference tube current-time product for this study is equal to $220 \mathrm{mAs}$, corresponding to a CTDI ${ }_{\text {vol }}$ equal to $34 \mathrm{mGy}$. However, because automatic exposure control was used, the mean effective tube current-time product was $90 \mathrm{mAs}$, corresponding to a $\mathrm{CTDI}_{\text {vol }}$ of $13.77 \mathrm{mGy}$.

examinations were acquired with detector collimation of $64 \times$ $0.6 \times 2 \mathrm{~mm}$ by using the $\mathrm{z}$-flying focal spot technique, ${ }^{7}$ gantry rotation time of 1 second, helical pitch of 0.6 , and tube potential of $120 \mathrm{kV}$. Automatic exposure control was used (CARE Dose4D; Siemens) with a quality reference tube current-time product equal to $220 \mathrm{mAs}$. The volume CT dose index $\left(\mathrm{CTDI}_{\mathrm{vol}}\right)$ for a standard-size patient when using this protocol is $34 \mathrm{mGy}$; however, because automatic exposure control was used, the effective tube current-time product and, therefore, the $\mathrm{CTDI}_{\mathrm{vol}}$ were much lower in the smaller pediatric head, as shown in Fig 2.

\section{Simulated Dose and Image Reconstruction}

Lower radiation dose CT datasets were simulated by using a validated method for inserting noise into the existing CT projection data acquired with routine parameters. The method incorporates the effect of the scanner bow-tie filter, automatic exposure control settings, and detector electronic noise. ${ }^{8}$ For each case, projection data with 3 simulated lower radiation dose levels were created, corresponding to $25 \%, 10 \%$, and $2 \%$ of the routine radiation dose (75\%, $90 \%$, and $98 \%$ radiation dose reduction). Axial and coronal images at routine and lower radiation doses were reconstructed by using 2.0-mm image thickness, 1.0-mm interval, 250-mm FOV, and routine head reconstruction kernel (J70), as shown in Fig 3. $3 \mathrm{D}$ volume-rendered images were created from axial images (image thickness, $0.75 \mathrm{~mm}$; interval, $0.70 \mathrm{~mm}$; FOV, $250 \mathrm{~mm}$; J30 kernel) by using 2 different shading methods (3D bone and 3D shining bone; Syngo VIA Version VA30 [Siemens]), as shown in Fig 4. All images were reconstructed using iterative reconstruction (SAFIRE; Siemens) with a strength parameter adapted to the radiation dose level (strengths 2, 3, 4, and 4 for routine, 25\%, 10\%, and $2 \%$ radiation-dose levels, respectively). 

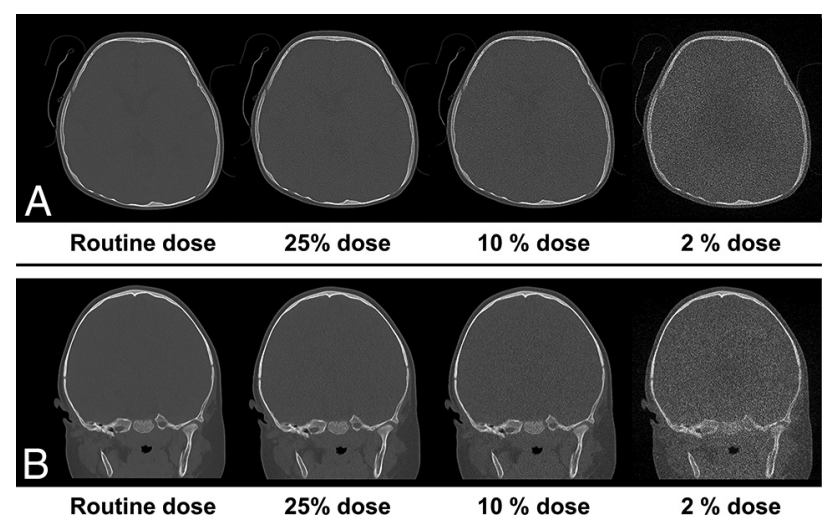

FIG 3. Axial and coronal images at routine dose and simulated lower radiation dose of a patient with left coronal craniosynostosis $(A)$ and sagittal craniosynostosis $(B)$.

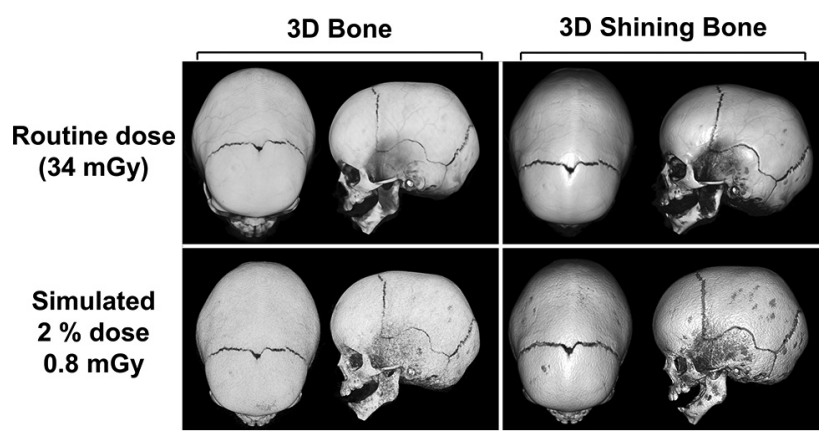

FIG 4. 3D volume-rendered images of a patient with sagittal craniosynostosis at routine dose and $2 \%$ routine dose, created from axial images (image thickness, $0.75 \mathrm{~mm}$; interval, $0.70 \mathrm{~mm}$; FOV, $250 \mathrm{~mm}$; J30 kernel) by using 2 different shading methods (3D bone and 3D shining bone).

\section{Image Evaluation}

Cases were anonymized, randomized, and divided into 4 reading sessions so that examinations from the same patient at each different radiation dose were reviewed in separate sessions, with a required minimum of 7 days between each session. Three neuroradiologists (D.R.D., A.L.K., F.E.D.), each with more than 7 years of experience, blinded to patient history and radiation dose level, evaluated images on a computer workstation (Advantage Windows Workstation; GE Healthcare, Milwaukee, Wisconsin) so that each patient at each radiation dose level was evaluated a single time by each reader. Each suture (sagittal, metopic, left coronal, right coronal, left lambdoid, right lambdoid, left squamosal, and right squamosal) was evaluated for craniosynostosis (positive, partial, or negative) by using $3 \mathrm{D}$ volume-rendered images. The $3 \mathrm{D}$ shading technique preference (3D bone, 3D shining bone, or no preference) and rationale for preference (image quality improvement versus difference in diagnosis) were noted for every case. Subsequently, readers evaluated 2D images (bone window, axial, and coronal) and 3D volume-rendered images together. For each suture evaluation, a diagnostic reader confidence score was determined by a 5 -point scale (1, negative without doubt; 3 , indeterminate; and 5, positive without doubt). After $2 \mathrm{D}$ and $3 \mathrm{D}$ images were evaluated together, an overall image quality score was recorded for every case at each radiation dose (1, nondiagnostic because of noise/artifacts; 2, diagnosis questionable because of noise/artifacts; 3 , diagnostic with moderate but acceptable noise/ artifacts; 4 , mild noise, no change in diagnostic confidence; and 5, routine diagnostic quality).

\section{Reference Standard}

The reference standard for craniosynostosis in each suture was defined by using reader agreement rules when $2 \mathrm{D}$ and $3 \mathrm{D}$ images were evaluated together at routine radiation dose. A suture was categorized as negative or positive if at least 2 of the 3 readers identified the suture as open versus fused/partially fused, respectively.

\section{Statistical Analysis}

Continuous variables were expressed as mean \pm SD or median and range. Categoric variables were expressed as frequencies and percentages. For every reader interpretation, the reference standard was compared with reader confidence score for each suture at each radiation dose. To account for multiple sutures per patient, we used a clustered receiver operating curve analysis with an ROI paradigm. ${ }^{9,10}$ A figure of merit (FOM), equal to the probability that the confidence of a randomly selected positive suture is greater than a randomly selected negative suture, was calculated for each reader at each radiation dose. For multireader analysis, readers were treated as fixed effects, and the average FOM was calculated across readers for each radiation dose. The difference between FOM and routine radiation dose was calculated and standard errors were estimated using the deformation-based morphometry method. ${ }^{11}$ Large-sample 95\% CIs, which are symmetrical about the point estimate, were estimated from the modelbased standard errors. Descriptive analyses and graphics were conducted by using SAS version 9.4 (SAS Institute, Cary, North Carolina). The RJafroc package (version 0.1 .1 ) on $\mathrm{R}$ version 3.1 (http://www.r-project.org) was used for estimation of the FOM and associated CIs.

\section{RESULTS}

\section{Study Population}

Thirty-three pediatric patients who underwent unenhanced CT of the head with an indication for craniosynostosis were included in the study. Five patients were scanned after surgery for question of recurrent or residual craniosynostosis. Patient ages ranged from neonate to 4 years old (median age, 8 months). The mean $\mathrm{CTDI}_{\mathrm{vol}}$ for the routine examination was $15.5 \pm 2.3 \mathrm{mGy}$ (range, 9.69-19.38 mGy), and the mean of the estimated effective dose when using age-corrected conversion factors ${ }^{12}$ was $1.96 \pm 0.48$ $\mathrm{mSv}$ (range, 1.09-3.2 mSv).

\section{Reference Standard}

According to reader agreement when $2 \mathrm{D}$ and $3 \mathrm{D}$ images were evaluated together at routine radiation dose, 21 patients had craniosynostosis for a total of 39 positive sutures and 225 negative sutures. Fifteen patients had single-suture craniosynostosis (7 sagittal, 5 metopic, 2 left coronal, and 1 right coronal), 1 patient had bilateral coronal craniosynostosis, and 5 patients had 3 or more closed sutures. 
Figures of merit and $95 \% \mathrm{Cl}$ for reader-specific and multireader evaluation of craniosynostosis using 3D images only and 2D images together with 3D images ${ }^{2}$

\begin{tabular}{|c|c|c|c|c|c|c|c|c|}
\hline & \multicolumn{2}{|c|}{ Routine } & \multicolumn{2}{|c|}{$25 \%$} & \multicolumn{2}{|c|}{$10 \%$} & \multicolumn{2}{|c|}{$2 \%$} \\
\hline & 3D Only & $2 D+3 D$ & 3D Only & $2 D+3 D$ & 3D Only & $2 D+3 D$ & 3D Only & $2 D+3 D$ \\
\hline Reader 1 & $0.89(0.83-0.96)$ & $0.86(0.78-0.94)$ & $0.94(0.88-1.00)$ & $0.92(0.87-0.98)$ & $0.91(0.85-0.97)$ & $0.87(0.75-0.98)$ & $0.90(0.82-0.97)$ & $0.88(0.80-0.96)$ \\
\hline Reader 2 & $0.93(0.88-0.98)$ & $0.94(0.90-0.99)$ & $0.92(0.87-0.97)$ & $0.88(0.82-0.94)$ & $0.86(0.77-0.95)$ & $0.88(0.80-0.97)$ & $0.85(0.76-0.94)$ & $0.85(0.75-0.94)$ \\
\hline Reader 3 & $0.95(0.92-0.99)$ & $0.95(0.92-0.98)$ & $0.90(0.84-0.97)$ & $0.89(0.82-0.95)$ & $0.92(0.87-0.98)$ & $0.93(0.88-0.98)$ & $0.85(0.69-1.00)$ & $0.84(0.68-1.00)$ \\
\hline Average & $0.92(0.90-0.95)$ & $0.91(0.89-0.95)$ & $0.92(0.88-0.97)$ & $0.89(0.86-0.94)$ & $0.89(0.84-0.95)$ & $0.89(0.83-0.96)$ & $0.86(0.79-0.94)$ & $0.85(0.76-0.95)$ \\
\hline
\end{tabular}

Data presented as FOM $(95 \% \mathrm{Cl})$.

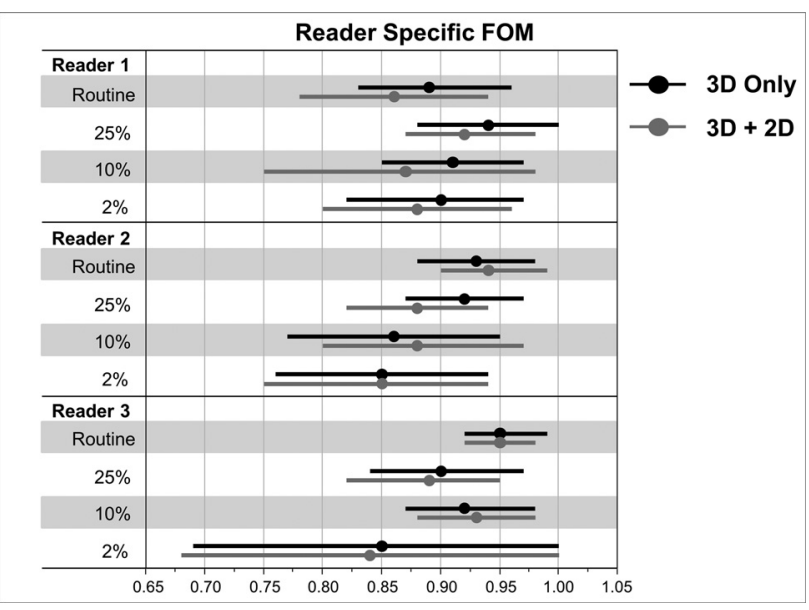

FIG 5. FOM and $95 \% \mathrm{Cl}$ for reader-specific evaluation of craniosynostosis by using 3D images only and 3D together with 2D images.

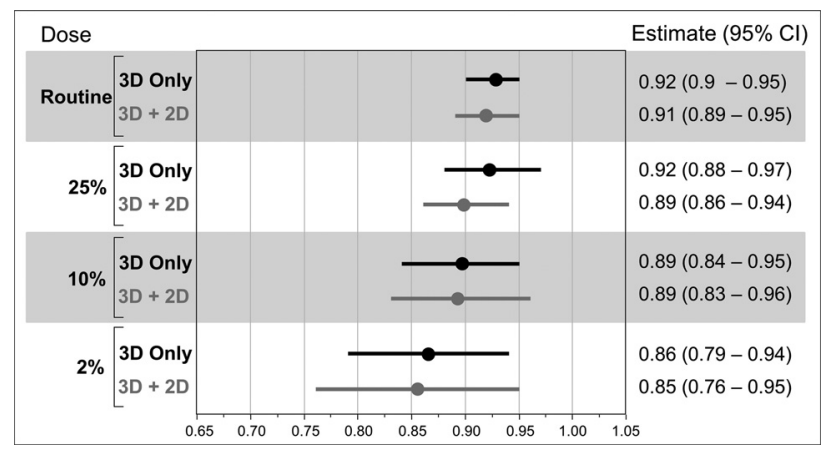

FIG 6. Average FOM and $95 \% \mathrm{CI}$ from all readers for all radiation dose levels in 3D and 3D together with 2D evaluation.

\section{Image Evaluation}

FOMs for reader-specific evaluation of craniosynostosis when using $3 \mathrm{D}$ images only and 2D and 3D images together are shown in the Table and Fig 5. Across all readers, performance estimates between $3 \mathrm{D}$ images and $2 \mathrm{D}$ and $3 \mathrm{D}$ images together were similar. For evaluation of 3D images only, pooled FOM for multireader analysis ranged from $0.92(95 \% \mathrm{CI}, 0.90-0.95)$ at routine dose to 0.86 (95\% CI, 0.79-0.94) at 2\% dose (Fig 6). Performance was similar when evaluating $2 \mathrm{D}$ and $3 \mathrm{D}$ images together, with pooled FOM ranging from 0.91 (95\% CI, 0.89-0.95) at routine dose to 0.85 (95\% CI, 0.76-0.95) at 2\% dose, as shown in the Table and Fig 6.

The estimated differences in FOM for the evaluation of craniosynostosis between the routine dose and the lower-dose configurations is shown in Fig 7. Minimum difference in FOM $(-0.01 ; 95 \% \mathrm{CI},-0.05$ to 0.04$)$ was achieved in the evaluation by using $3 \mathrm{D}$ images only at $25 \%$ radiation dose. At $2 \%$ radiation dose, performance in the evaluation was degraded, with a FOM

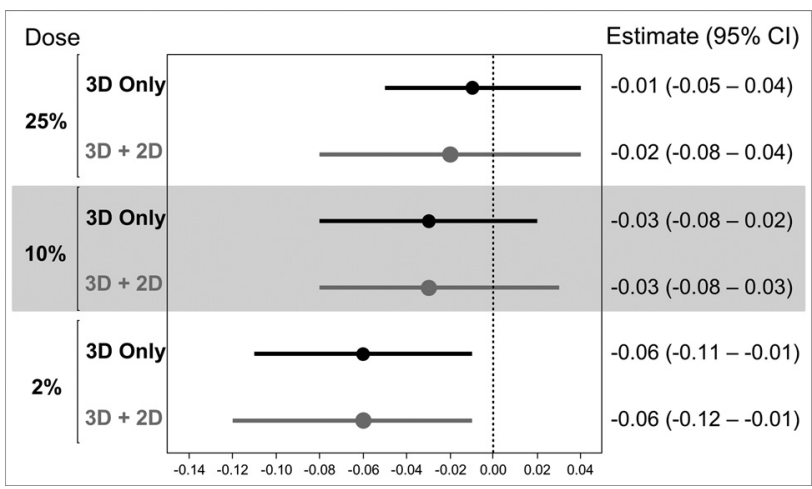

FIG 7. Difference in FOM (with $95 \% \mathrm{Cl}$ ) between each radiation dose level and reference standard in $3 D$ and $3 D$ together with $2 D$ evaluation.

difference between routine and 2\% dose of -0.06 ( $95 \% \mathrm{CI},-0.12$ to -0.01 ) for both $3 \mathrm{D}$ only and $2 \mathrm{D}$ and $3 \mathrm{D}$ images together, so this lower-dose configuration is considered inferior.

\section{D Volume-Rendering Preference and Image Quality}

For all readers and radiation doses, 3D shining bone was preferred in 270 of 396 evaluations (68\%). There was no preference in 117 evaluations (30\%), and the 3D bone setting was preferred in only 7 (2\%) of the evaluated conditions. Rationale for 3D volumerendered preference was improved image quality in 222 of 270 (82\%) evaluations when 3D shining bone was preferred and, similarly, in 5 of 7 (71\%) evaluations when 3D bone was preferred. Better visualization of sutures with impact in diagnosis was chosen as rationale for preference in 48 of 270 (18\%) evaluations when 3D shining bone was preferred and 2 of $7(29 \%)$ evaluations when $3 \mathrm{D}$ bone was preferred.

Image quality scores for each reader and radiation dose are summarized in Fig 8. For all readers and radiation doses, the percentage of cases rated as having diagnostic image quality (score $\geq 3$ ) were $100 \%, 98 \%, 96 \%$, and $78 \%$ for routine, $25 \%, 10 \%$, and $2 \%$ radiation dose levels, respectively.

\section{DISCUSSION}

Pediatric patients with suspected craniosynostosis often need CT for diagnosis and planning of management. These scans are performed to evaluate the status of sutures to determine whether a head deformity is caused by positional plagiocephaly or premature sutural closure, known as craniosynostosis. ${ }^{13}$ For most patients, however, surgery is the primary treatment. ${ }^{1}$ To help confirm diagnosis and guide surgical treatment, imaging is frequently obtained, usually in the form of CT.

Using a validated method to simulate lower radiation dose CT examinations, reduced dose scans were compared with images acquired using our clinical standard of care. Our study demon- 


\section{Radiation Dose Level}

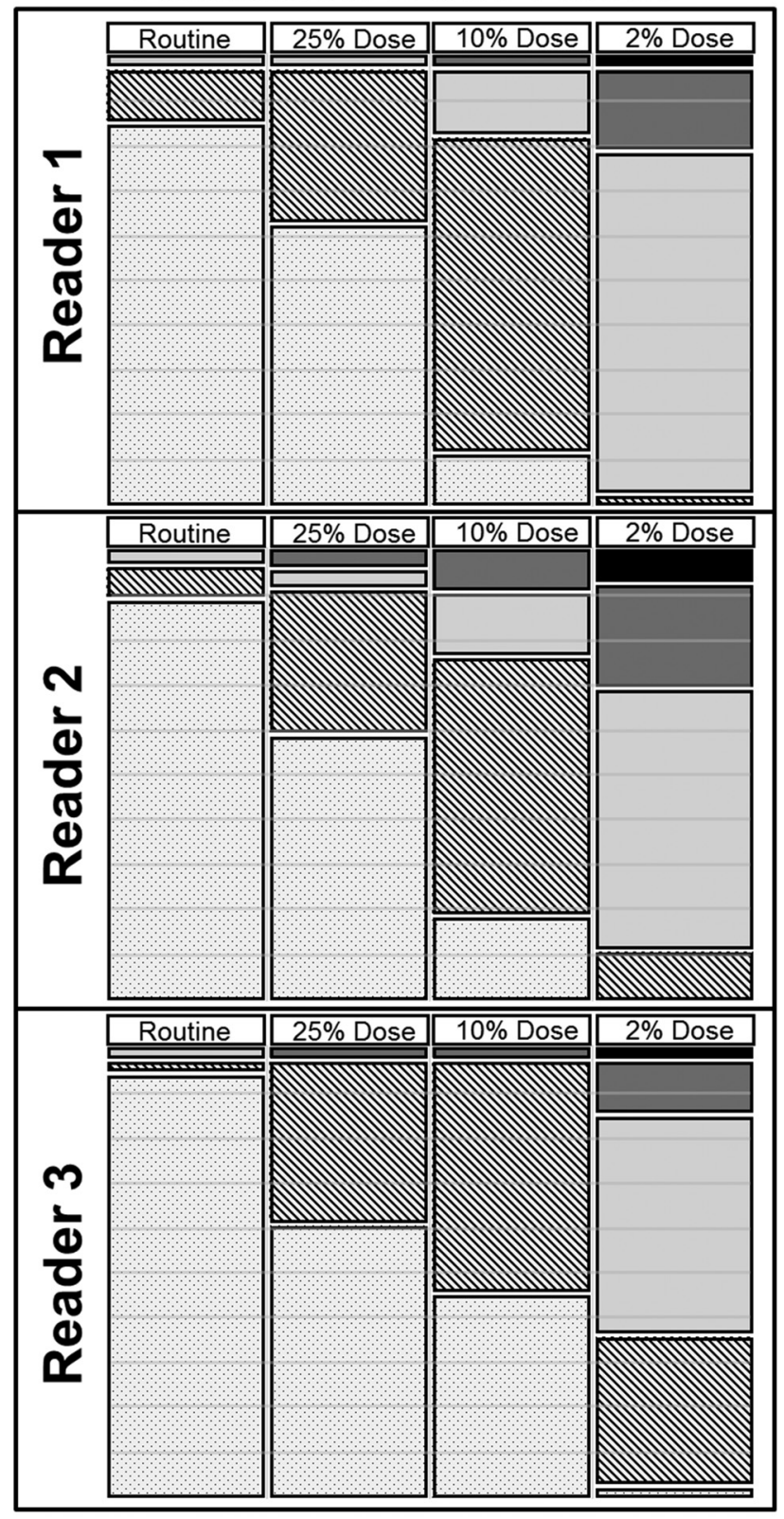

Overall Image Quality Score

$1=$ Non-diagnostic due to noise/artifacts

$2=$ Diagnosis questionable due to noise/artifacts

3= Diagnostic with moderate but acceptable noise/artifacts

4h = Mild Noise, no change in diagnostic confidence $5=$ Routine diagnostic quality

FIG 8. Stacked percent bar plot of image quality scores for each reader and radiation dose.

strated that a $75 \%-90 \%$ reduction in dose relative to a routine head CT examination was clinically acceptable for the evaluation of craniosynostosis. In this blinded study, neuroradiologists were able to accurately detect craniosynostosis across all dose levels (25\%, $10 \%$, and $2 \%$ of the routine radiation dose) without compromising diagnostic performance. As expected, the overall image quality decreased at lower doses; however, this did not compromise the diagnostic accuracy for this specific indication when the reference $\mathrm{CTDI}_{\mathrm{vol}}$ was reduced from $34 \mathrm{mGy}$ to a simulated value of $3.8 \mathrm{mGy}$ (approximately $10 \%$ of routine radiation dose). Even at the $2 \%$ dose, moderate noise or artifacts were noted, but images were still acceptable for diagnosis in more than $80 \%$ of the cases. In all cases, 3D volume-rendered images were used with both $3 \mathrm{D}$ bone and 3D shining bone techniques. When using 3D images compared with $2 \mathrm{D}$ and $3 \mathrm{D}$ images together, reader performance was similar.

Others have investigated ways to reduce radiation exposure in patients with suspected craniosynostosis, and our work is complementary and adds to their efforts. In 2015, Kaasalainen et $\mathrm{al}^{14}$ published data concluding that dose reductions over $80 \%$ were possible for CT evaluation of craniosynostosis. However, their analysis was performed on phantoms with simplified cranial anatomy. Our study has shown that similar dose reductions are possible in actual patients with various anatomic appearances. In addition, Ernst et $\mathrm{al}^{15}$ reported in 2016 that a dedicated sub-0.1 $\mathrm{mSv}$ cranial 3D CT protocol can be used without loss in image quality, but this study did not report radiologist performance, only image quality. In their study, patients were scanned with either routine or low-dose CT protocols, so only image quality was assessed, and there was no radiologist assessment of suture closure or comparison with a reference standard. Our comparison of matched cases across a range of CT doses permitted paired comparisons of radiology diagnoses across radiation doses. Finally, Morton et al ${ }^{16}$ described their successful institutional experience using low-dose head CT in various pediatric conditions, though their study was not limited to craniosynostosis, and lowdose images were not directly compared with full-dose images from the same patient at the same time. Alternatively, some authors have proposed ultrasonographic evaluation of cranial sutures in children with suspected craniosynostosis, but that approach has not yet gained widespread acceptance, perhaps in part because children with positive findings at sonography are still recommended to undergo CT for preoperative evaluation, and falling doses used in CT evaluation of craniosynostosis diminishes the need for a zero-dose technique that has not yet demonstrated superiority to $\mathrm{CT} .^{17}$

There were several limitations to our study. This was a pilot retrospective study intended to provide an initial estimate of how dose reduction and reconstruction approaches could be used synergistically to maintain diagnostic performance. There was a relatively small number of cases, evaluated by 3 readers. Reader agreement rules were applied to the interpretation of routinedose CT images for determination of the reference standard. We decided to use the actual reader interpretations rather than design a separate consensus interpretation to better illustrate that small disagreements in the evaluation did not substantially affect the diagnostic conclusion that dose reductions are possible regardless of the individual reader. Moreover, in the context of this study, we believe this approach actually inflates the observer performance at routine dose while potentially increasing the difference in FOMs relative to the reduced-dose images. Although this was not considered ideal from a pure efficacy approach, it was selected as a pragmatic approach to this pilot study. Because of mild differences in technique, blinded readers were likely able to distinguish the original-dose studies from the reconstructed lower-dose stud- 
ies at $10 \%$ and $2 \%$ of the original dose. This could have resulted in bias during subjective image quality evaluation, despite the readers being blinded to technical data. In addition, the design of our questionnaire may have prompted the blinded radiologists to look more closely for craniosynostosis than they would have without prompting, and associated intracranial abnormalities were not evaluated. Finally, implementation of $10 \%$ - and $25 \%$-dose scanning for craniosynostosis can result in high accuracy, but does not adequately evaluate underlying brain structures; MR or routine-dose CT must be used if this additional diagnostic task is necessary.

Despite these limitations, our study demonstrates sufficient diagnostic performance and image quality of low-dose CT for the task of evaluating craniosynostosis.

\section{CONCLUSIONS}

The accuracy of detecting craniosynostosis was maintained across all dose levels by using appropriate strengths of iterative reconstruction. As expected, image quality mildly to moderately decreased at lower-dose levels, but without compromise in diagnostic performance down to dose reductions on the order of $90 \%$. Tailored low-dose CT examinations for the evaluation of craniosynostosis are a reasonable method to decrease the radiation associated with CT while minimizing risk and addressing concerns that might result in detrimental deferral of diagnostic imaging.

Disclosures: Cynthia H. McCollough—RELATED: Grant. Siemens Healthcare*; UNRELATED: Grants/Grants Pending: Siemens Healthcare.* Joel G. Fletcher-UNRELATED: Grants/ Grants Pending: Siemens Healthcare, Comments: grant support for CT Clinical Innovation Center. * Money paid to the institution.

\section{REFERENCES}

1. Kirmi O, Lo SJ, Johnson D, et al. Craniosynostosis: a radiological and surgical perspective. Semin Ultrasound CT MR 2009;30:492-512 CrossRef Medline

2. Bristol RE, Lekovic GP, Rekate HL. The effects of craniosynostosis on the brain with respect to intracranial pressure. Semin Pediatr Neurol 2004;11:262-67 CrossRef Medline

3. Schweitzer T, Böhm H, Meyer-Marcotty P, et al. Avoiding CT scans in children with single-suture craniosynostosis. Childs Nerv Syst 2012;28:1077-82 CrossRef Medline

4. McCollough CH, Bushberg JT, Fletcher JG, et al. Answers to com- mon questions about the use and safety of CT scans. Mayo Clin Proc 2015;90:1380-92 CrossRef Medline

5. Gabriel S, Eckel LJ, DeLone DR, et al. Pilot study of radiation dose reduction for pediatric head $\mathrm{CT}$ in evaluation of ventricular size. AJNR Am J Neuroradiol 2014;35:2237-42 CrossRef Medline

6. Ehman EC, Yu L, Manduca A, et al. Methods for clinical evaluation of noise reduction techniques in abdominopelvic CT. Radiographics 2014;34:849-62 CrossRef Medline

7. Flohr TG, Stierstorfer K, Ulzheimer S, et al. Image reconstruction and image quality evaluation for a 64-slice CT scanner with z-flying focal spot. Med Phys 2005;32:2536-47 CrossRef Medline

8. Yu L, Shiung M, Jondal D, et al. Development and validation of a practical lower-dose-simulation tool for optimizing computed tomography scan protocols. J Comput Assist Tomogr 2012;36:477-87 CrossRef Medline

9. Obuchowski NA. Nonparametric analysis of clustered ROC curve data. Biometrics 1997;53:567-78 CrossRef Medline

10. Obuchowski NA, Lieber ML, Powell KA. Data analysis for detection and localization of multiple abnormalities with application to mammography. Acad Radiol 2000;7:516-25 CrossRef Medline

11. Dorfman DD, Berbaum KS, Metz CE. Receiver operating characteristic rating analysis. Generalization to the population of readers and patients with the jackknife method. Invest Radiol 1992;27: 723-31 CrossRef Medline

12. American Association of Physicists in Medicine. The Measurement, Reporting, and Management of Radiation Dose in CT: Report of AAPM Task Group 23 of the Diagnostic Imaging Council Committee. College Park, Maryland: American Association of Physicists in Medicine; 2008. AAPM report no. 96

13. Nagaraja S, Anslow P, Winter B. Craniosynostosis. Clin Radiol 2013; 68:284-92 CrossRef Medline

14. Kaasalainen T, Palmu K, Lampinen A, et al. Limiting CT radiation dose in children with craniosynostosis: phantom study using modelbased iterative reconstruction. Pediatr Radiol 2015;45:1544-53 CrossRef Medline

15. Ernst CW, Hulstaert TL, Belsack D, et al. Dedicated sub 0.1 mSv 3DCT using MBIR in children with suspected craniosynostosis: quality assessment. Eur Radiol 2016;26:892-99 CrossRef Medline

16. Morton RP, Reynolds RM, Ramakrishna R, et al. Low-dose head computed tomography in children: a single institutional experience in pediatric radiation risk reduction. J Neurosurg Pediatrics 2013;12:406-10 CrossRef

17. Simanovsky N, Hiller N, Koplewitz B, et al. Effectiveness of ultrasonographic evaluation of the cranial sutures in children with suspected craniosynostosis. Eur Radiol 2009;19:687-92 CrossRef Medline 